О СЕМАНТИЧЕСКОЙ МОДЕЛИ ПРЕДМЕТНОЙ ОБЛАСТИ «УРАВНЕНИЯ МАТЕМАТИЧЕСКОЙ ФИЗИКИ» ${ }^{1}$

Тучкова Наталия Павловна

$$
\text { к.ф.-м.н., с.н.с., }
$$

Вычислительный центр им. А.А. Дородницына

Федерального исследовательского центра «Информатика и управление»

Российской академии наук, e-mail: natalia_tuchkova@mail.ru,

ORCID [0000-0001-6518-5817],

г. Москва, 119333, ул. Вавилова, 40

\begin{abstract}
Аннотация. Анализируются связи понятий математической предметной области на примере раздела уравнений математической физики. Предлагается вариант статьи тезауруса для терминов и связанных с ними уравнений и формул. Особенность такого тезауруса заключается в использовании контекста формул для их дополнительной идентификации в предметной области. Кроме того, предлагается учитывать индексы авторов и статей, где встречаются термины тезауруса. Предложенный подход способствует уточнению поискового запроса и уменьшению информационного шума при использовании тезауруса в цифровых библиографических коллекциях.
\end{abstract}

Ключевые слова: тезаурус предметной области, уравнения смешанного типа, поисковый запрос.

Цитирование: Тучкова Н. П. О семантической модели предметной области «Уравнения математической физики»//Информационные и математические технологии в науке и управлении. 2020. № 4 (20). C. 132-142. DOI: 10.38028/ESI.2020.20.4.012

Введение. Применение тезаурусов в современных цифровых ресурсах является повсеместным средством семантического описания коллекций. Опыт создания лингвистических тезаурусов довольно длинный, начиная от тезауруса английского языка Роже (Roget's Thesaurus) [26], который переиздается уже более 150 лет. Актуальность этого процесса повысилась с появлением языков онтологий консорциума W3C $[21,28]$. Технология онтологического проектирования, по определению, позволяет непосредственно воспроизвести структуру и связи, содержащиеся в тезаурусе, средствами программирования [20]. Многочисленные примеры прикладных онтологий и инструментов редактирования данных появились около 2003 года, когда были опубликованы первые рекомендации W3C, в частности, тезаурусы английского языка 21 века [25, 27]. В работе [6] представлена современная модель лингвистической онтологии на основе информационно-поискового тезауруса [5, 18, 22] на примере русскоязычных ресурсов. Опыт создания прикладных тезаурусов по отдельным предметным областях гораздо меньше. Это диктуется спецификой такой работы, которая подразумевает совместные исследования в области информационных

\footnotetext{
${ }^{1}$ Работа выполнена при частичной поддержке Российского фонда фундаментальных исследований, проект № 18-29-10085мк
} 
технологий и в предметной области. Целый ряд прикладных тезаурусов был создан в 80-е годы прошлого столетия в период расцвета отечественной кибернетики $[1,4,8,11-13,15,17]$. В это же время появился результат работы ведущих ученых-математиков - математическая энциклопедия под редакцией академика И.М. Виноградова [7]. Далее наступила эпоха оцифровки знаний [3] и эта советская энциклопедия стала основой для представления математических знаний в Интернет, где она реализована в русском ${ }^{2}$ и английском ${ }^{3}$ вариантах, а также как один из источников информации в системе LibMeta 4 .

В настоящей работе развивается онтологический подход к представлению математических знаний в цифровых ресурсах применительно к разделам смешанных задач математической физики.

1. Математические знания в Интернет. Представление в Интернет математических знаний - одна из актуальных задач информационных технологий в силу востребованности математики, как в образовании, так и в научных исследованиях. Особенности 21 столетия и дистанционного образования в период пандемии COVID-19 обозначили необходимость достоверных оцифрованных знаний, в том числе и математических. Это не явилось неожиданностью для научного сообщества, поскольку оно уже давно, начиная с первых разработок канадского математика из университета Торонто Кеннета Мэй (Kenneth O. May), занималось систематизацией знаний. Этому ученому принадлежит авторство уникального цифрового проекта по созданию словарей и тезаурусов по математике совместно с издательством McGrow-Hill в 70-е годы прошлого столетия. Тезаурус на основе терминологии математических заметок (Mathematics Notes) и других журналов был буквально собран группой студентов на библиотечных карточках. Современные разработки в этой области относятся, конечно, к использованию онтологического представления словарей и тезаурусов в базах данных и цифровых библиографических коллекциях.

Особенности информационных образов математических предметных областей в контексте задач математической физики были проанализированы в работе [10], там же приведены ссылки на некоторые открытые источники с таблицами иерархических структур и родовидовых отношений в тех случаях, когда это явно представлено в самих источниках. Важным качеством математических и многих других естественно-научных текстов является наличие формул. Математическая формула выступает как свертка информации, которая может быть использована и в описании вторичного документа, и в поисковом образе, также может быть помещена в соответствующую статью словаря или тезауруса. Математическая формула представляет собой уникальный объект предметной области, который несет в себе информацию независимо от языка изложения материала. Это стало поводом для разработки методов использования этой информации в информационно-поисковых системах научных публикаций. Вторичный документ формируется как результат аналитико-синтетической переработки информации. Свертывание информации - это совокупность операций аналитикосинтетической переработки, преследующих цель выразить содержание текста в более короткой форме при сохранении или некотором уменьшении информативности в производном тексте [2, 16]. В качестве сверток информации могут выступать законы, теоремы,

\footnotetext{
${ }^{2}$ https://dic.academic.ru/contents.nsf/enc_mathematics

${ }^{3}$ https://encyclopediaofmath.org/wiki/Main_Page

${ }^{4}$ http://libmeta.ru/
} 
формулы и тому подобные обобщения, концентрирующие в себе «свертывание» огромного фактического материала.

Одно из первых появлений формул в тезаурусе относится к химии ${ }^{5}$, что вполне оправдано и стало возможным после появления современных средств визуализации. В русскоязычном сегменте в 1972 г. появился «Тезаурус научно-технических наук» [15], в 1978 г. «Тезаурус по теории формальных и алгебраических групп» [4], в 1991 г. «Русско-немецкий информационно-поисковый тезаурус по робототехнике»[1]. В 2005 г. был опубликован «Тезаурус информационно-поисковый по предметной области: обыкновенные дифференциальные уравнения» [9], где были даны основные дескрипторы и связи терминов в этой области. В дальнейшем этот тезаурус был интегрирован в семантическую библиотеку LibMeta $^{6}$.

Можно отметить Zentralblatt MATH (https://zbmath.org, Mathematical abstracts and reviews), как информационный ресурс, наиболее адекватно отвечающий запросам пользователей математических библиотек, использующих формулы, как в представлении публикаций, так и в информационном запросе, Здесь содержатся данные о публикациях, начиная с 1826 года по настоящее время. Цель его создания обозначена как сбор, систематизация и распространение библиографических данных, рефератов книг и статей, посвящённых всем разделам математики и её приложениям. В частности, этот проект свидетельствует о том, что интерес к проблеме использования символьной записи в виде формул в поисковом запросе сформировался достаточно давно. Zentralblatt MATH демонстрирует на своей библиографической базе данных преимущества использования символьной информации, формул. Надо отметить, что аналогичный интегрированный проект для отечественных публикаций пока не реализован, хотя ведется много работ в этом направлении. Это - «Общероссийский математический портал» (http://www.mathnet.ru/) математического института им. В.А Стеклова РАН; Интернет-проект (http://eqworld.ipmnet.ru/indexr.htm) «Мир математических уравнений» (EqWorld); pecypc «OntoMathpro Ontology classes» (http://ontomathpro.org/ontology) Казанского университета, содержащий англо-русский алфавитный указатель терминов с горизонтальными связями, и многие другие.

В направлении интеграции математических ресурсов на основе онтологического представления развивается семантическая библиотека LibMeta, в которую наряду с другими источниками интегрированы математическая энциклопедия, тезаурус обыкновенных дифференциальных уравнений, классификаторы MSC, УДК и др. Расширение предметной области математической физики реализуется через увеличение связей и понятий отдельных разделов, в частности для смешанных задач [14].

2. Структура статьи тезауруса для смешанных уравнений математической физики. Уравнения смешанного типа являются частью предметной области уравнений в частных производных (УЧП) и принимают гиперболический тип (hyperbolic type, HT), параболический тип (parabolic type, PT) и эллиптический тип (elliptic type, ET), в зависимости от области определения (рассмотрения), как часть УЧП (схема на рис. 1).

На рис. 1 схематически показаны несколько уровней родовидовых отношений для УЧП

\footnotetext{
5 http://www.wssanalytchem.org/ontology/default.aspx
}

${ }^{6} \mathrm{http}: / /$ libmeta.ru/thesaurus/show/19115 
второго порядка с независимыми переменными в самом общем виде. Эта предметная область содержит практически необъятный материал, что затрудняет поиск публикаций конкретной тематики.

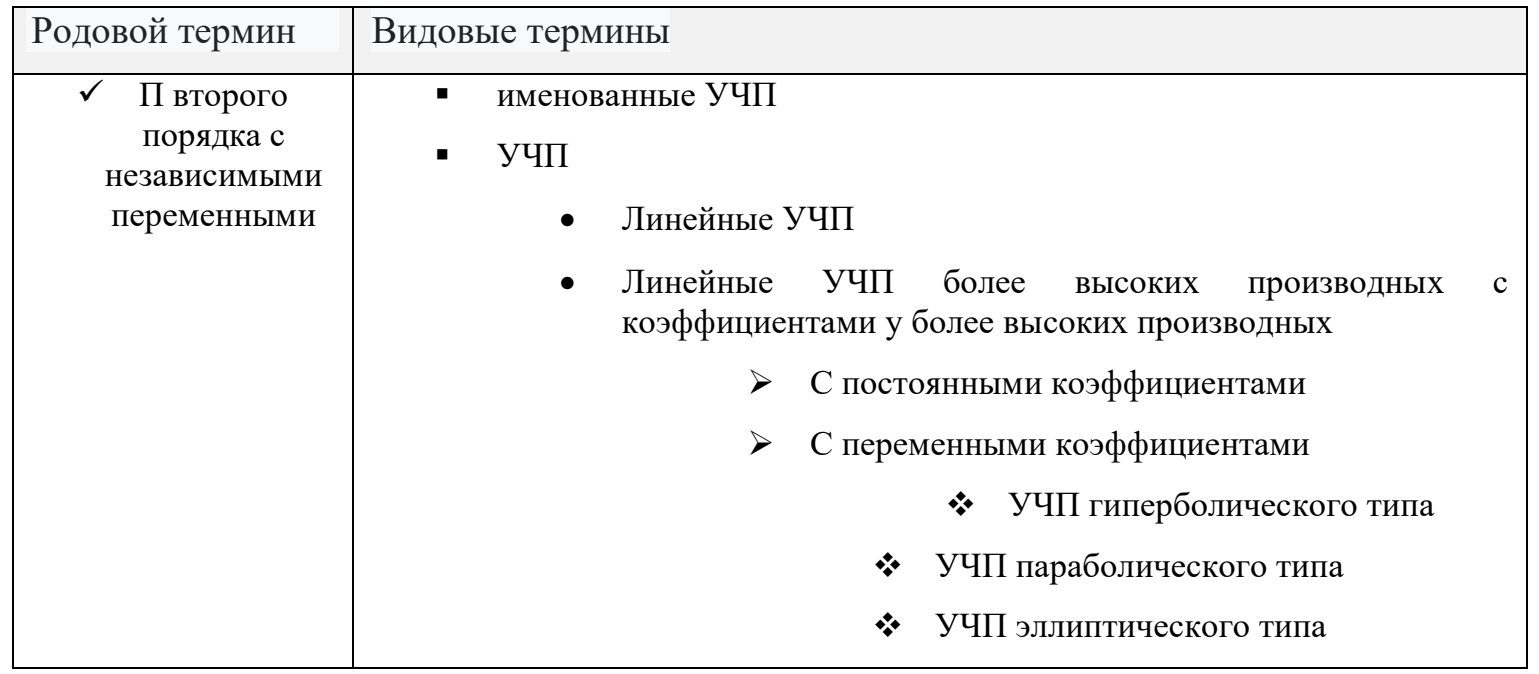

Рис. 1. Схема родовидовых связей УЧП второго порядка с независимыми переменными

Чем больше признаков можно использовать в поисковом запросе, тем точнее можно осуществить поиск, поэтому состав статьи тезауруса необходимо расширить для того, чтобы было возможно использовать различные точки входа, как минимум, название, автор и формула. Например, для уравнения Трикоми, одного из самых изучаемых в теории смешанных уравнений, статья тезауруса представлена в таблице 1.

Таблица 1. Статья тезауруса для дескриптора «Трикоми уравнение»

\begin{tabular}{|c|c|c|}
\hline $\begin{array}{l}\text { Позиции } \\
\text { статьи } \\
\text { тезауруса }\end{array}$ & $\begin{array}{l}\text { Тип } \\
\text { данных, } \\
\text { язык }\end{array}$ & Значения позиций статьи тезауруса \\
\hline PDE1031(MXT) & $\mathrm{Ru}$ & Трикоми уравнение \\
\hline & En & Tricomi equation \\
\hline Син1 & $\mathrm{Ru}$ & Уравнение Т \\
\hline Син2 & En & Equation $\mathrm{T}$ \\
\hline РодMSC & $35 \mathrm{M} 10$ & Equations of mixed type \\
\hline РодУДК & 517.956 .6 & Уравнения смешанного типа \\
\hline ВидФорм 1 & MSWord & $y \frac{\partial^{2} u}{\partial x^{2}}+\frac{\partial^{2} u}{\partial y^{2}}=0$ \\
\hline ВидФорм2 & LaTex & $\$ y \mid$ frac $\left\{\mid\right.$ partial $\left.{ }^{\wedge} 2 \mathrm{u}\right\}\left\{\backslash\right.$ partial $\left.\mathrm{x}^{\wedge} 2\right\}+\mid$ frac $\left\{\mid\right.$ partial $\left.{ }^{\wedge} 2 \mathrm{u}\right\}\left\{\mid\right.$ partial $\left.\mathrm{y}^{\wedge} 2\right\}=0 \$$ \\
\hline ВидФорм3 & LaTex & $\$ y u_{-}\{x x\}+u_{-}\{y y\}=0 \$$ \\
\hline Лит1 & {$[1 \mathrm{ru}]$} & Смирнов М. М. Уравнения смешанного типа. М., 1970. 296 с. \\
\hline Лит2 & {$[2 \mathrm{ru}]$} & $\begin{array}{l}\text { Трикоми Ф. Лекции по уравнениям в частных производных. Пер. с итал. } \\
\text { Д. А. Райкова с предисл. Б. М. Левитана М.: Изд-во иностр. лит., 1957. } 443 \text { с. }\end{array}$ \\
\hline Лит3 & [1en] & $\begin{array}{l}\text { Smirnov M.M. Equations of mixed type. American Translation of the Mathematical } \\
\text { monografs. Vol. 51. Mathematical Soc., } 31 \text { Dec 1978. p: } 232 .\end{array}$ \\
\hline Лит4 & [2en] & $\begin{array}{l}\text { Lupo D., Morawetz C.S., Payne K.R. On closed boundary value problems for } \\
\text { equations of mixed elliptic-hyperbolic type // Communications on Pure and Applied } \\
\text { Mathematics, 2007, } 60 \text { (9), 1319-1348 }\end{array}$ \\
\hline Род & $\mathrm{Ru}$ & $\mid$ PDE1030(МХT)| Уравнения смешанного типа \\
\hline Род & En & |PDE1030(MXT)| Equations of mixed type \\
\hline Вид 1 & & PDE1031(CF2,HT,y<0) \\
\hline Вид 2 & & $\mid$ PDE1031(CF,ET, $\mathrm{y}>0) \mid$ \\
\hline Вид 3 & & $\left|\mathrm{PDE} 1031\left(\mathrm{CF}, \mathrm{ET}, z=\eta^{\alpha} u, \alpha=-1 / 6\right)\right|$ \\
\hline Вид 4 & & $\left|\mathrm{PDE} 1031\left(\mathrm{CF}, \mathrm{ET}, z=\eta^{\alpha} u, \alpha=-1 / 3\right)\right|$ \\
\hline
\end{tabular}


Тучкова Н. П.

\begin{tabular}{|l|l|l|}
\hline КлС & $\begin{array}{l}\text { Трикоми задача, обобщение Трикоми задачи, Трикоми метод, задача Коши } \\
\text { для уравнения Трикоми, краевая задача для уравнения типа Чаплыгина, } \\
\text { краевая задача для уравнения смешанного типа }\end{array}$ \\
\hline Acc & $\mathrm{Ru}$ & $|\mathrm{PDE} 1037(\mathrm{MXT})|$ Геллерстедта задача \\
\hline Acc & $\mathrm{En}$ & $|\mathrm{PDE} 1037(\mathrm{MXT})|$ The Gellerstedt problem \\
\hline См. также & & $\mid$ PDE1039(МХT)| Газовой динамики уравнение смешанного типа \\
\hline История & & $\begin{array}{l}\text { Название термина по имени Трикоми Франческо Джакомо (Tricomi Francesco } \\
\text { Giacomo), появилось в [1ru] как ссылка на работу[2ru] }\end{array}$ \\
\hline Примечания & & $\mid$ PDE1036(МХT)| Геллерстедта решение задачи Трикоми для уравнения \\
\hline
\end{tabular}

В Таблице 1 PDE1031(MXT)| - это мнемонический идентификатор дескриптора, где PDE - сокращение от Partial Differential Equations (уравнения в частных производных), MXT - сокращение от Mixed Туре (смешанный тип), 1031 - номер дескриптора в цифровой библиотеке.

Позиции статьи тезауруса включают родовые, видовые и ассоциативные термины. В качестве видовых добавлены формулы, в качестве родовых - классификаторы. По сравнению со стандартными полями тезауруса ГОСТ [5] добавлены следующие: См. также, История, Примечания. Все эти поля не противоречат стандартам, поскольку могут быть отнесены к ассоциативным терминам и для наглядности вынесены в отдельные позиции.

Основные дескрипторы именных уравнений смешанного типа - это «Трикоми уравнение» и «Геллерстедта задача». Для одного из них выписаны ключевые слова в Таблице 2 со списком публикаций, где они встречаются в Таблице 3.

Таблица 2. Ключевые слова дескриптора «Геллерстедта задача»

\begin{tabular}{|c|c|c|}
\hline 1 & $\begin{array}{l}\text { уравнение для Геллерстедта задачи }[2,3 \text {, } \\
4,5,7,8,10,11,12,13,14,15,16,17,18,19,20]\end{array}$ & $\begin{array}{l}\text { - equation for Gellerstedt problem } \\
\text { - problem G }\end{array}$ \\
\hline 2 & уравнение типа Чаплыгина & $\begin{array}{l}\text { - equations of Chaplygin's type } \\
\text { - Chaplygin type equation }\end{array}$ \\
\hline 3 & $\begin{array}{l}\text { уравнение смешенного типа[2, } 3 \text {, } \\
4,5,7,8,10,11,12,13,14,15,16,17,18,19,20]\end{array}$ & - equations of mixed type \\
\hline 4 & Трикоми задача $[10,12,14,15,16,19,20]$ & $\begin{array}{l}\text { - Tricomi problem } \\
\text { - problem T }\end{array}$ \\
\hline 5 & обобщение Трикоми задачи $[10,12,14,15,16,19,20]$ & $\begin{array}{l}\text { - generalized Tricomi problem } \\
\text { - generalized problem T }\end{array}$ \\
\hline 6 & Трикоми метод $[10,12,14,15,16,19,20]$ & $\begin{array}{l}\text { - Tricomi's method } \\
\text { - Tricomi method }\end{array}$ \\
\hline 7 & $\begin{array}{l}\text { задача Коши для уравнения Трикоми } \\
{[10,12,14,15,16,19,20]}\end{array}$ & - Cauchy's problem for Tricomi's equation \\
\hline 8 & краевая задача для уравнения типа Чаплыгина & - boundary-value problem Chaplygin type equation \\
\hline 9 & $\begin{array}{l}\text { краевая задача для уравнения смешанного типа }[2, \\
3,4,5,7,8,10,11,12,13,14,15,16,17,18,19,20]\end{array}$ & $\begin{array}{l}\text { - boundary-value problem for a mixed- } \\
\text { type equation }\end{array}$ \\
\hline 10 & $\begin{array}{l}\text { эллиптичность уравнения для Геллерстедта задачи } \\
{[2,3,4,5,7,8,10,11,12,13,14,15,16,17,18,19,20]}\end{array}$ & - ellipticity of the equation for Gellerstedt problem \\
\hline 11 & $\begin{array}{l}\text { параболичность для уравнения Геллерстедта задачи } \\
{[2,3,4,5,7,8,10,11,12,13,14,15,16,17,18,19,20]}\end{array}$ & $\begin{array}{l}\text { - parabolicity of the equation for Gellerstedt } \\
\text { problem }\end{array}$ \\
\hline 12 & $\begin{array}{l}\text { гиперболичность для уравнения Геллерстедта } \\
\text { задачи }[2,3,4,5,7,8,10,11,12,13,14,15,16,17,18, \\
19,20]\end{array}$ & $\begin{array}{l}\text { - hyperbolicity of the equation for Gellerstedt } \\
\text { problem }\end{array}$ \\
\hline
\end{tabular}




\begin{tabular}{|c|l|l|}
\hline 13 & $\begin{array}{l}\text { теорема существования и единственности для } \\
\text { уравнения Геллерстедта задачи }[2,3, \\
4,5,7,8,10,11,12,13,14,15,16,17,18,19,20]\end{array}$ & $\begin{array}{l}\text { - existence and uniqueness theorem for the } \\
\text { equation for Gellerstedt problem }\end{array}$ \\
\hline 14 & $\begin{array}{l}\text { теорема существования и единственности для } \\
\text { обобщенной задачи Трикоми }\end{array}$ & $\begin{array}{l}\text { - existence and uniqueness theorems for the } \\
\text { generalized Tricomi problem }\end{array}$ \\
\hline 15 & $\begin{array}{l}\text { метод Геллерстедта [2, 3, 4,5,7,8,10,11,12,13, } \\
14,15,16,17,18,19,20]\end{array}$ & - Gellerstedt's method \\
\hline 16 & уравнение газовой динамики смешанного типа & - gaz dinamycal equation of mixed type \\
\hline 17 & $\begin{array}{l}\text { приложения уравнения Геллерстедта задачи в } \\
\text { околозвуковой газовой динамике }\end{array}$ & $\begin{array}{l}\text { - applications of the Gellerstedt problem in } \\
\text { transsonic gas dynamics }\end{array}$ \\
\hline 18 & $\begin{array}{l}\text { уравнение для Геллерстедта задача смешанного } \\
\text { типа с оператором Лаврентьева-Бицадзе [2, 3, } \\
4,5,7,8,10,11,12,13,14,15,16,17,18,19,20]\end{array}$ & $\begin{array}{l}\text { - Gellerstedt problem for the mixed type equation } \\
\text { with Lavrentyev-Bisadze operator } \\
\text { - mixed type problem G with LB operator }\end{array}$ \\
\hline
\end{tabular}

Таблица 3. Литература к ключевым словам по теме «Геллерстедта задача» из таблицы 2.

\begin{tabular}{|c|c|}
\hline 1 & $\begin{array}{l}\text { Математическая Энциклопедия. Т. } 1 \text { (А - Г). Ред. коллегия: И. М. Виноградов (глав. ред.) [и др.] - М., } \\
\text { «Советская Энциклопедия», 1977, } 1152 \text { стб. с илл. }\end{array}$ \\
\hline 2 & Gellerstedt S. Doctoral Thesis, 1935; Jbuch Fortschritte Math. 61, 1259. \\
\hline 3 & Rassias J.M. Lecture Notes on Mixed Type Partial Differential Equations. World Scientific, 1990, 144 p. \\
\hline 4 & $\begin{array}{l}\text { Трикоми Ф.Д., Лекции по уравнениям в частных производных, пер. с итал., М.:Изд-во иностранной } \\
\text { литературы, 1957, } 446 \text { с. }\end{array}$ \\
\hline 5 & Смирнов М. М. Уравнения смешанного типа. М., 1970. 296 с. \\
\hline 6 & $\begin{array}{l}\text { Smirnov M.M. Equations of mixed type. American Translation of the Mathematical monografs. Vol. } 51 . \\
\text { Mathematical Soc., } 31 \text { Dec 1978. p: } 232 .\end{array}$ \\
\hline 7 & $\begin{array}{l}\text { Моисеев Е.И., Таранов Н.О. Решение одной задачи Геллерстедта для уравнения Лаврентьева- } \\
\text { Бицадзе // Дифференциальные уравнения. 2009. Т. 45, № 4. С. 543-548. }\end{array}$ \\
\hline 8 & $\begin{array}{l}\text { Моисеев Е.И., Таранов Н.О. Интегральное представление решения одной задачи Геллерстедта // } \\
\text { Дифференциальные уравнения. 2009. Т. 45, № 11. С. 1554-1559. }\end{array}$ \\
\hline 9 & $\begin{array}{l}\text { Моисеев Е.И., Лихоманенко Т.Н. Об одной нелокальной краевой задаче для уравнения Лаврентьева- } \\
\text { Бицадзе // Доклады Академии наук. 2012. Т. 446, № 3. С. 256-258. }\end{array}$ \\
\hline 10 & $\begin{array}{l}\text { Moiseev E.I., Nefedov P.V. Tricomi problem for the lavrent'ev-bitsadze equation in a 3d domain // Integral } \\
\text { Transforms and Special Functions. 2012. Vol. 23, no. 10. P. 761-768. }\end{array}$ \\
\hline 11 & $\begin{array}{l}\text { Moiseev E.I., Nefedov P.V. Gellerstedt problem for the Lavrent'ev-Bitsadze equation in a 3D-domain // } \\
\text { Integral Transforms and Special Functions, V. 25, Issue 7, 2014 DOI: 10.1080/10652469.2014.883618. }\end{array}$ \\
\hline 12 & $\begin{array}{l}\text { Моисеев Е.И., Холомеева А.А., Нефедов П.В. Аналоги задач Трикоми и Франкля в трехмерных } \\
\text { областях для уравнения Лаврентьева-Бицадзе // Дифференциальные уравнения, том 50, № 12, } 2014 . \\
\text { C. } 1672-1675 . \text { DOI: } 10.1134 / \text { S0374064114120127 }\end{array}$ \\
\hline 13 & $\begin{array}{l}\text { Moiseev E.I., Nefedov P.V., Kholomeeva A.A. Analog of the Gellerstedt problem for the Lavrent'ev- } \\
\text { Bitsadze equation in a 3D domain Differential Equations // Differential Equations издательство МAIK } \\
\text { Nauka/Interpereodika Publishing (Russian Federation), 2015. Т. 51, № 6. С. 827-829. }\end{array}$ \\
\hline 14 & $\begin{array}{l}\text { Moiseev E.I., Moiseev T.E., Vafodorova G.O. On an Integral Representation of Neumann-Tricomi Problem } \\
\text { for the Lavrent'ev-Bitsadze Eguation // Differential Equations, MAIK (Russian Federation), 2015, T. 51, № } \\
\text { 8, C. 1065-1071. }\end{array}$ \\
\hline 15 & $\begin{array}{l}\text { Моисеев Е.И., Лихоманенко Т.Н. Собственные функции задачи Трикоми с наклонной линией } \\
\text { изменения типа // Дифференциальные уравнения. 2016. Т. 52, № 10, С. 1375-1382. }\end{array}$ \\
\hline 16 & $\begin{array}{l}\text { Zarubin A.N., Kholomeeva A.A. Tricomi problem for an advance-delay equation of mixed type with } \\
\text { variable deviation of the argument // Differential Equations. 2016. Vol. 52, no. 10. P. 1312-1322. }\end{array}$ \\
\hline 17 & $\begin{array}{l}\text { Моисеев Е.И., Моисеев Т.Е., Холомеева А.А. О Разрешимости задачи Геллерстедта с данными на } \\
\text { параллельных характеристиках // Дифференциальные уравнения. 2017. Т. 53, № 10. С. 1379-1384. }\end{array}$ \\
\hline 18 & $\begin{array}{l}\text { Moiseev E.I., Likhomanenko T.N. Eigenfunctions of the gellerstedt problem with an inclined-type change } \\
\text { line // Integral Transforms and Special Functions. 2017. Vol. 28, no. 4. P. 328-335. }\end{array}$ \\
\hline 19 & $\begin{array}{l}\text { Moiseev E.I., Likhomanenko T.N. Eigenfunctions of the tricomi problem with an inclined type change line } \\
\text { // Differential Equations. 2016. Vol. 52, no. 10. P. 1323-1330. }\end{array}$ \\
\hline 20 & $\begin{array}{l}\text { Moiseev E.I., Gulyaev D.A. The completeness of the eigenfunctions of the tricomi problem for the } \\
\text { Lavrent'ev-Bitsadze equation with the Frankl gluing condition // Integral Transforms and Special } \\
\text { Functions. 2016. Vol. 27, no. 11. P. 893-898. }\end{array}$ \\
\hline
\end{tabular}


В Таблицах 2 и 3 содержатся данные, которые входят в соответствующие позиции статей тезауруса (пример - таблица 1). Для краткости изложения они вынесены здесь в отдельные таблицы и не снабжены идентификаторами. Все позиции Таблицы 2 связаны видовыми отношениями с главным термином, дескриптором «Геллерстедта задача», а сам термин «Геллерстедта задача» связан ассоциативными связями с термином-дескриптором «Трикоми уравнение».

Ключевые слова также выступают в роли контекстов формул в поисковом запросе.

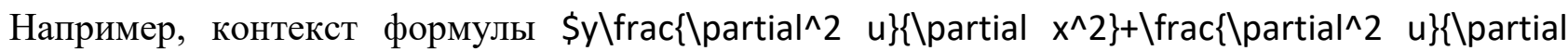

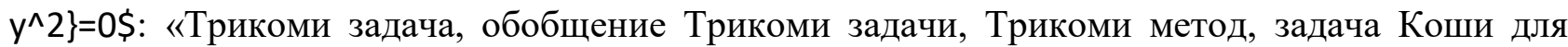
уравнения Трикоми, краевая задача для уравнения типа Чаплыгина, краевая задача для уравнения смешанного типа» из таблицы 1 и их английские эквиваленты из таблицы 2. Кроме того, контекстом могут служить ключевые слова из реферативного списка таблицы 3 , а также индексы авторов (ORCID и другие). Таким образом, используя связи тезауруса, можно уточнять или расширять поисковый запрос, достигая в результате уменьшение информационного шума.

Заключение и выводы. В работе рассмотрены примеры формирования статей информационно-поискового тезауруса для одного из разделов предметной области задач математической физики. Сама предметная область охватывает широкий класс задач, поэтому выбран конкретный раздел, на примере которого можно в дальнейшем распространить онтологическое описание на другие разделы. Данное представление статей тезауруса не противоречит основному математическому ресурсу, математической энциклопедии И.М. Виноградова, а делается в интеграции с ней в рамках одной семантической библиотеки LibMeta [19]. Необходимость дополнения и расширения оцифрованной математической энциклопедии обусловлена тем, что этот цифровой вариант ограничен реферативными ссылками 80-х годов, когда эта энциклопедия была опубликована. Дальнейшая работа предполагается в направлении развития онтологического представления математических знаний в семантических ресурсах и расширения предметной области информационного запроса в соответствии с новыми методами информационных технологий.

\section{СПИСОК ЛИТЕРАТУРЫ}

1. Барт К., Мицевич А.Т., Петрина А.М., Риглер Ш., Сукачева Е.В., Тош В., Хадзиламбру 3.Н. Русско-немецкий информационно-поисковый тезаурус по робототехнике. М.: ВИНИТИ. 1991. 318 с.

2. Блюменау Д.И. Проблемы свертывания научной информации. Л.:Наука. 1982.166 с.

3. Гаврилова Т.А. Хорошевский В.Ф. Базы знаний интеллектуальных систем. СПб.: Питер. 2000. 384 c.

4. Глазунов, Н.М., Дриянский, В.М., Комарова, Т.Н., Рыбалко, Ю.А. Тезаурус по теории формальных и алгебраических групп и его реализация на ЕС ЭВМ. АН УССР. Ин-т кибернетики. 1978.39 с.

5. ГОСТ 7.25.-2001. Тезаурус информационно-поисковый одноязычный: Правила разработки: структура, состав и форма представления // Система стандартов по информации, библиотечному и издательскому делу. Минск: Межгосударственный совет по стандартизации, метрологии и сертификации. 2001. ГОСТ Р 7.0.91-2015 (ИСО 25964- 
1:2011) Система стандартов по информации, библиотечному и издательскому делу. Тезаурусы для информационного поиска.

6. Лукашевич Н.В., Добров Б.В. Проектирование лингвистических онтологий для информационных систем в широких предметных областях // Онтология проектирования. 2015. T. 5. №. 1 (15). С.47-69.

7. Математическая энциклопедия. Гл. ред. И.М. Виноградов. М.: Советская энциклопедия. 1977-1985. Тома 1-5. 1104 с.

8. Мищенко Г.А., Калинина Г.Р., Гладкова Г.И., Губенко Н.Т., Джигирханова А.В. Двуязычный информационно-поисковый тезаурус классов органических соединений (русско-немецкий/немецко-русский). Пер. на немецкий язык Dr. H.-J.Tepper. М.:ВИНИТИ. 1987. 384 с.

9. Моисеев Е.И., Муромский А.А., Тучкова Н.П. Тезаурус информационно-поисковый по предметной области: обыкновенные дифференциальные уравнения. М.:МАКС Пресс. 2005. $116 \mathrm{c}$.

10. Муромский А.А., Тучкова Н.П. Представление математических понятий в онтологии научных знаний // Онтология проектирования. 2019. Т. 9. № 1 (31). С. 50- 69. DOI:10.18287/2223-9537-2019-9-1-50-69.

11. Никитина С.Е. Тезаурус по теоретической и прикладной лингвистике (автоматическая обработка текста). М.:Наука. 1978. 375 с.

12. Пащенко Н.А., Ксенофонтова Е.Б., Скоробогатая В.Ф. Информационно-поисковый тезаурус по информатике. М.: ВИНИТИ. 1987. 466 с.

13. Рождественский Ю.В. Словарь терминов: общеобразовательный тезаурус: Общество. Семиотика. Экономика. Культура. Образование. М.: Флинта: Наука. 2002. 112 с.

14. Смирнов М.М. Уравнения смешанного типа. М. 1970. 296 с.

15. Тезаурус научно-технических наук / Под.ред. Ю.И. Шемакина. М.: Воениздат. 1972. $671 \mathrm{c}$.

16. Шрейдер Ю.А. Об одной модели семантической теории информации // Проблемы кибернетики. М.: Наука. 1965. Вып. 18. С. 233-240.

17. Шрейдер Ю.А. Тезаурусы в информатике и теоретической семантике // Научнотехническая информация. Сер. 2. 1971. № 3. С. 21-24.

18. ANSI/NISO Z39.19-2005, Guidelines for the Construction, Format, and Management of Monolingual Controlled Vocabularies. Bethesda. MD: NISO Press. 2005.

19. Ataeva O., Serebryakov V., Tuchkova N. Query Expansion Method Application for Searching in Mathematical Subject Domains // CEUR Workshop Proceedings, M. Jeusfeld c/o Redaktion Sun SITE, Informatik V, RWTH Aachen (Germany). 2020. V. 2543. Pp. 38-48. Available at: http://ceur-ws.org/Vol-2543/rpaper04.pdf urn:nbn:de:0074-2543-4 (accessed 06.12.2020)

20. Berners-Lee T., Handler J., Lassila O. The Semantic Web // Scientific American 2001. V. 284. № 5. P. 28-37.

21. Gomez-Perez A., Corcho O. Ontology languages for the Semantic Web // IEEE Intelligent Systems. 2002. Vol. 17. № 1. P. 54-60. DOI: 10.1109/5254.988453

22. ISO 25964-1:2011. Thesauri and interoperability with other vocabularies. Part 1: Thesauri for information retrieval / Geneva: International Organization for Standards. 2011. 
23. May K.O. Historiography: A Perspective for Computer Scientists. Invited address to International Research Conference on the History of Computing. 1976. Los Alamos.

24. Niles I., Pease A. Linking Lexicons and Ontologies: Mapping WordNet to the Suggested Upper Merged Ontology // Proceedings of the IEEE International Conference on Information and Knowledge Engineering. 2003. P. 412-416.

25. Prinseton university. A Lexical Database for English Available at: https://wordnet.princeton.edu/ (accessed 06.12.2020)

26. Roget P.M. Roget's thesaurus of English words and phrases. Taylor Anderson (Editor). CreateSpace Independent Publishing Platform. 2017. 660 p.

27. Thesaurus.com. Available at: https://www.thesaurus.com/ (accessed 06.12.2020)

28. World Wide Web Consortium (W3C). Available at: https://www.w3.org (accessed 06.12.2020)

\title{
UDK 004.89
}

\author{
ON SEMANTIC MODEL OF SUBJECT DOMAIN \\ "MATHEMATICAL PHYSICS EQUATIONS" \\ Natalia P. Tuchkova \\ $\mathrm{PhD}$., senior researcher, \\ Dorodnicyn Computing Center \\ of Federal Research Center "Informatics and Control" \\ of Russian Academy of Science, \\ e-mail: natalia_tuchkova@mail.ru, \\ ORCID [0000-0001-6518-5817] \\ Vavilov st. 40, 119333 Moscow, Russia
}

\begin{abstract}
The studying is focusing to the relationship of the concepts of the mathematical subject area on the example of the section of mathematical physics equations. A version of the thesaurus article for terms and related equations and formulas is proposed. The peculiarity of such a thesaurus lies in the use of the context of formulas for their additional identification in the subject area. In addition, it is proposed to take into account the indices of authors and articles where thesaurus terms are found. This approach helps to refine the search query and reduce information noise when using the thesaurus in digital bibliographic collections.
\end{abstract}

Keywords: subject domain thesaurus, equations of mixed type, search query.

\section{References}

1. Bart K., Mitsevich A.T., Petrina A.M., Rigler SH., Sukacheva Ye.V., Tosh V., Khadzilambru Z.N. Russko-nemetskiy informatsionno-poiskovyy tezaurus po robototekhnike [Russian-German information retrieval thesaurus on robotics]. M.: VINITI = M.: All-Russian Institute of Scientific and Technical Information. 1991. 318p.

2. Blyumenau D.I. Problemy svertyvaniya nauchnoy informatsii [Collapsing scientific information]. L.: Nauka=L.: Science. 1982. 166p. 
3. Gavrilova T.A. Khoroshevskiy V.F. Bazy znaniy intellektual'nykh system [Knowledge base of intelligent systems]. SPb.: Piter= SPb.: Piter. 2000. 384p.

4. Glazunov, N.M., Driyanskiy, V.M., Komarova, T.N., Rybalko, YU.A. Tezaurus po teorii formal'nykh i algebraicheskikh grupp i yego realizatsiya na YES EVM [Thesaurus on the theory of formal and algebraic groups and its implementation on an ES computer]. AN USSR. In-t kibernetiki. 1978. 39p.

5. GOST 7.25.-2001. Tezaurus informatsionno-poiskovyy odnoyazychnyy: Pravila razrabotki: struktura, sostav i forma predstavleniya [Monolingual information retrieval thesaurus: Development rules: structure, composition and form of presentation] // Sistema standartov po informatsii, bibliotechnomu i izdatel'skomu delu. Minsk: Mezhgosudarstvennyy sovet po standartizatsii, metrologii i sertifikatsii = System of standards for information, librarianship and publishing. Minsk: Interstate Council for Standardization, Metrology and Certification. 2001. GOST R 7.0.91-2015 (ISO 25964-1:2011).

6. Lukashevich N.V., Dobrov B.V. Proyektirovaniye lingvisticheskikh ontologiy dlya informatsionnykh sistem $\mathrm{v}$ shirokikh predmetnykh oblastyakh [Designing linguistic ontologies for information systems in broad subject areasъ // Ontologiya proyektirovaniya= Design Ontology. 2015. V. 5. № 1 (15). P.47-69.

7. Matematicheskaya entsiklopediya. Gl. red. I.M. Vinogradov [Mathematical encyclopedia]. M.: Sovetskaya entsiklopediya =Soviet encyclopedia. 1977-1985. V. 1-5. 1104p.

8. Mishchenko G.A., Kalinina G.R., Gladkova G.I., Gubenko N.T., Dzhigirkhanova A.V. Dvuyazychnyy informatsionno-poiskovyy tezaurus klassov organicheskikh soyedineniy (russkonemetskiy/nemetsko-russkiy)[ Bilingual information retrieval thesaurus of classes of organic compounds (Russian-German / German-Russian)]/ Per. na nemetskiy yazyk Dr. H.-J.Tepper. M.:VINITI= M.:All-Russian Institute of Scientific and Technical Information. 1987.384p.

9. Moiseyev Ye.I., Muromskiy A.A., Tuchkova N.P. Tezaurus informatsionno-poiskovyy po predmetnoy oblasti: obyknovennyye differentsial'nyye uravneniya [Information retrieval thesaurus in the subject area: ordinary differential equations]. M.:MAKS Press= M.: MAX Press. 2005. 116p.

10. Muromskiy A.A., Tuchkova N.P. Predstavleniye matematicheskikh ponyatiy v ontologii nauchnykh znaniy [Representation of mathematical concepts in the ontology of scientific knowledge] // Ontologiya proyektirovaniya= Ontology of design. 2019.Vol. 9. № 1 (31). Pp. 5069. doi.org/10.18287/2223-9537-2019-9-1-50-69.

11. Nikitina S.Ye. Tezaurus po teoreticheskoy i prikladnoy lingvistike (avtomaticheskaya obrabotka teksta)[ Theoretical and Applied Linguistics thesaurus (automatic text processing)]. M.:Nauka= M.: Science. 1978. 375p.

12. Pashchenko N.A., Ksenofontova Ye.B., Skorobogataya V.F. Informatsionno-poiskovyy tezaurus po informatike[Information retrieval thesaurus in computer science]. M.: VINITI= M.: All-Russian Institute of Scientific and Technical Information. 1987.466p.

13. Rozhdestvenskiy YU.V. Slovar' terminov: obshcheobrazovatel'nyy tezaurus: Obshchestvo. Semiotika. Ekonomika. Kul'tura. Obrazovaniye [Glossary of terms: general education thesaurus: Society. Semiotics. Economy. Culture. Education]. M.: Flinta: Nauka = M .: Flinta: Science. 2002. 112 p.

14. Smirnov M.M. Uravneniya smeshannogo tipa [Mixed type equations]. M. 1970. 296p. 
15. Tezaurus nauchno-tekhnicheskikh nauk [Thesaurus of Scientific and Technical Sciences]. M.: Voyenizdat $=$ Moscow: Military Publishing. 1972.671p.

16. Shreyder YU.A. Ob odnoy modeli semanticheskoy teorii informatsii [On one model of semantic information theory]// Problemy kibernetiki= Problems of Cybernetics. M.: Nauka = M.: Science. 1965. Issue 18. Pp.233-240.

17. Shreyder YU.A. Tezaurusy $\mathrm{v}$ informatike i teoreticheskoy semantike // Nauchnotekhnicheskaya informatsiya [Thesauri in informatics and theoretical semantics] // Nauchnotekhnicheskaya informatsiya. Ser. 2. = Scientific and technical information. Ser. 2. 1971. № 3. P.21-24.

18. ANSI/NISO Z39.19-2005, Guidelines for the Construction, Format, and Management of Monolingual Controlled Vocabularies. Bethesda. MD: NISO Press. 2005.

19. Ataeva O., Serebryakov V., Tuchkova N. Query Expansion Method Application for Searching in Mathematical Subject Domains // CEUR Workshop Proceedings, M. Jeusfeld c/o Redaktion Sun SITE, Informatik V, RWTH Aachen (Germany). 2020. V. 2543. p. 38-48. Available at: http://ceur-ws.org/Vol-2543/rpaper04.pdf urn:nbn:de:0074-2543-4 (accessed 06.12.2020)

20. Berners-Lee T., Handler J., Lassila O. The Semantic Web // Scientific American 2001. V.284. № 5. P. 28-37.

21. Gomez-Perez A., Corcho O. Ontology languages for the Semantic Web // IEEE Intelligent Systems. 2002. Vol. 17. № 1. P. 54-60. DOI:10.1109/5254.988453

22. ISO 25964-1:2011. Thesauri and interoperability with other vocabularies. Part 1: Thesauri for information retrieval / Geneva: International Organization for Standards. 2011.

23. May K.O. Historiography: A Perspective for Computer Scientists. Invited address to International Research Conference on the History of Computing. 1976. Los Alamos.

24. Niles I., Pease A. Linking Lexicons and Ontologies: Mapping WordNet to the Suggested Upper Merged Ontology // Proceedings of the IEEE International Conference on Information and Knowledge Engineering. 2003. P. 412-416.

25. Prinseton university. A Lexical Database for English Available at: https://wordnet.princeton.edu/ (accessed 06.12.2020)

26. Roget P.M. Roget's thesaurus of English words and phrases. Taylor Anderson (Editor). CreateSpace Independent Publishing Platform. 2017. 660 p.

27. Thesaurus.com. Available at: https://www.thesaurus.com/ (accessed 06.12.2020)

28. World Wide Web Consortium (W3C). Available at: https://www.w3.org (accessed 06.12.2020) 